\title{
Efeitos de programas baseados em mindfulness oferecidos pela internet para pacientes com câncer: revisão integrativa de ensaios clínicos randomizados.
}

\author{
Effects of web-based mindfulness programs for cancer patients: integrative review of randomized \\ clinical trials.
}

Efectos de los programas de atención plena basados en Internet para pacientes con cáncer: revisión integradora de ensayos clínicos aleatorizados.

\author{
Daniela Maria Xavier de Souza \\ ORCID: https://orcid.org/0000-0002-7136-5934 \\ Universidade Federal de São Carlos, Brasil \\ E-mail: danielaxaviersouza@gmail.com \\ Ledjane Neves de Oliveira Schaeffer Greve \\ ORCID: https://orcid.org/0000-0003-4771-3051 \\ Universidade Federal de São Carlos, Brasil \\ E-mail: ledyneves@hotmail.com \\ Mônica Jordão de Souza Pinto \\ ORCID: https://orcid.org/0000-0003-0144-6680 \\ Universidade Federal de São Carlos, Brasil \\ E-mail: monijordao@hotmail.com \\ Marcelo Demarzo \\ ORCID: https://orcid.org/0000-0002-7447-1839 \\ Universidade Federal de São Paulo, Brasil \\ E-mail: marcelodemarzo@gmail.com \\ Priscilla Hortense \\ ORCID: https://orcid.org/0000-0003-0554-451X \\ Universidade Federal de São Carlos, Brasil \\ E-mail: priscillaufscar@gmail.com
}

\begin{abstract}
Resumo
Objetivo: Analisar ensaios clínicos randomizados que avaliaram os efeitos de intervenções baseadas em mindfulness oferecidas pela internet, como tratamento complementar para pacientes com diferentes tipos de câncer. Método: Foram construídas estratégias de buscas específicas para cada base de dados, com descritores controlados e não controlados, operadores booleanos e truncamentos. As buscas para seleção dos estudos foram realizadas nas bases de dados: Pubmed/Medline, PsycoInfo, Web of Science e Clinicaltrials.gov. Resultados: Foram incluídos 8 estudos na revisão integrativa, 2 destes ainda estão em desenvolvimento. Participaram dos estudos finalizados 714 indivíduos com diagnóstico de câncer de quaisquer tipo ou localização. As intervenções online baseadas em mindfulness avaliadas nos estudos incluídos, evidenciaram efeitos positivos em desfechos relevantes para saúde psicológica dos pacientes, tais como ansiedade, depressão, humor, sono, qualidade de vida, estresse, fadiga, crescimento póstraumático, bem-estar, espiritualidade. Conclusão: Intervenções baseadas em mindfulness por meio da internet tem se mostrado terapias promissoras para a promoção da saúde com foco na qualidade de vida e redução do sofrimento psicológico.
\end{abstract}

Palavras-chave: Atenção plena; Neoplasias; Intervenção baseada em internet.

\begin{abstract}
Objective: To analyze randomized clinical trials evaluating the effects of mindfulness-based processes offered over the internet, as a complementary treatment for patients with different types of cancer. Method: Specific search strategies were built for each database, with controlled and uncontrolled descriptors, Boolean operators and truncations. Searches for study selection were performed in the following databases: Pubmed / Medline, PsycoInfo, Web of Science and Clinicaltrials.gov. Results: 8 studies were included in the integrative review, 2 of which are still under development. A total of 714 specialists with a diagnosis of cancer of any type or location participated in the completed studies. Online investigations based on mindfulness evaluated in the included studies showed positive effects on relevant outcomes for patients' psychological health, such as anxiety, depression, mood, sleep, quality of life, stress, fatigue, post-traumatic growth, well-being, spirituality. Conclusion: Interventions based on mindfulness through the internet have if there are promising therapies for health promotion with a focus on quality of life and reduction of psychological distress.
\end{abstract}

Keywords: Mindfulness; Neoplasms; Internet-based intervention. 


\begin{abstract}
Resumen
Objetivo: Analizar ensayos clínicos aleatorizados que evalúen los efectos de los procesos basados en mindfulness ofrecidos a través de Internet, como tratamiento complementario para pacientes con diferentes tipos de cáncer. Método: Se construyeron estrategias de búsqueda específicas para cada base de datos, con descriptores controlados y no controlados, operadores booleanos y truncamientos. Las búsquedas para la selección de estudios se realizaron en las siguientes bases de datos: Pubmed / Medline, PsycoInfo, Web of Science y Clinicaltrials.gov. Resultados: Se incluyeron 8 estudios en la revisión integradora, 2 de los cuales aún se encuentran en desarrollo. En los estudios terminados participaron un total de 714 especialistas con diagnóstico de cáncer de cualquier tipo o localización. Las investigaciones en línea basadas en la atención plena evaluadas en los estudios incluidos mostraron efectos positivos en resultados relevantes para la salud psicológica de los pacientes, como ansiedad, depresión, estado de ánimo, sueño, calidad de vida, estrés, fatiga, crecimiento postraumático, bienestar, espiritualidad. Conclusión: Las intervenciones basadas en la atención plena a través de Internet tienen si existen terapias prometedoras para la promoción de la salud con un enfoque en la calidad de vida y la reducción de la angustia psicológica.
\end{abstract}

Palabras clave: Atención Plena; Neoplasias; Intervención basada en la Internet.

\title{
1. Introdução
}

Em março de 2020 a COVID-19, como é chamada a doença causada pelo novo coronavírus SARS-COV-2, foi considerada uma pandemia com 118.000 casos em 114 países (WHO 2021). No que se refere às pessoas com câncer, além das adaptações requeridas por este novo contexto somam-se o medo, as preocupações com dores e desconfortos, as sequelas de tratamentos, o risco de progressão e recorrência da doença, os atrasos no diagnóstico e tratamento (Ueda et al., 2020), assim, estas pessoas podem evoluir para um sistema imunológico ainda mais enfraquecido estando mais susceptíveis a infecções (Mehta et al., 2020; Kuderer et al., 2020). Considerando as adequações necessárias a este novo cenário, é importante se atentar às necessidades das pessoas com câncer buscando mitigar os impactos desta pandemia em suas vidas (Young et al., 2020).

Em tempos de pandemia, uma maneira de minimizar estes efeitos deletérios na vida das pessoas é por meio da internet visto que a necessidade de distanciamento social imposto favorece intervenções que possam ocorrer de forma remota. O oferecimento de serviços de saúde mental pela internet traz algumas vantagens, como a garantia de uma maior privacidade, a possibilidade de ser acessível a pessoas de diferentes lugares ao mesmo tempo, constante atualização de conhecimentos científicos e baixo custo comparado aos tratamentos presenciais, possibilitando ainda que seu conteúdo seja traduzido para outras línguas, atingindo maior diversidade de pessoas (Leykin et al., 2011). Trevino et al (2020) descreve uma ampla utilização e satisfação dos participantes com a implantação de serviços mente-corpo fornecidos remotamente para pessoas com câncer durante o COVID-19.

Os programas baseados em mindfulness ou MBIs (Mindfulness Based Interventions) têm sido propostos para manejo da variedade de fatores que compõem a experiência do adoecimento e tratamento do câncer, trazendo resultados promissores em inúmeras pesquisas realizadas nas últimas décadas (Kubo et al., 2020; Lengacher et al., 2019; Metin, et al., 2019). A prática de mindfulness pode trazer uma redução na fadiga relacionada ao câncer (He et al., 2020), benefícios como melhora da dor, do estresse, da ansiedade e da depressão (Ngamkham, Holden, \& Smith, 2019), redução do sofrimento psicológico, do medo de recorrência do câncer e melhora do sono (Cillensen et al., 2019), assim como maior índice de qualidade de vida, crescimento pós-traumático e atenção plena (Xunlin, Lau \& Klainin-Yobas (2020).

Um ensaio clínico destinado a pacientes com dor crônica propôs comparar modos de entrega de uma MBI, observando que tanto a entrega presencial como por videoconferência trouxera ganhos semelhantes em saúde mental e na redução da catastrofização da dor em relação aos controles (Gardner-Nix et al., 2008). Carlson et al (2019) aponta a relevância de pesquisas com programas de mindfulness entregues por meio de aplicativos, garantindo que pacientes que não possam comparecer em programas guiados online recebam uma "dose" menor de treinamento (Carlson et al., 2019). Desta forma, o objetivo desta revisão integrativa foi analisar os efeitos de MBIs oferecidos via internet, seja no modo síncrono guiado por um instrutor, ou de forma assíncrona ou por meio de aplicativos ou websites, para pessoas com diferentes tipos de câncer. 


\section{Método}

Esta é uma revisão integrativa de ensaios clínicos randomizados (ECRs) que foi guiada pelo Preferred Reporting items for Systematic Review and meta-analyses (Prisma) (Liberati et al., 2009).

A revisão foi baseada na seguinte questão de pesquisa: "Quais os efeitos de uma intervenção baseada em mindfulness (MBI) na internet para adultos que tiveram diagnóstico de câncer?"

\section{Critérios de inclusão e exclusão dos estudos}

Foram incluídos ensaios clínicos randomizados com intervenções baseadas em mindfulness na internet, de maneira síncrona ou assíncrona, que estudaram adultos com diagnóstico de câncer.

Os estudos inseridos nesta revisão não tiveram restrições de idioma e não foi estabelecido limite temporal, evitando restringir demasiadamente a busca e ampliando a abrangência para recuperar publicações potencialmente relevantes.

Estudos que incluíram como participantes parceiros e cuidadores, estudos quase-randomizados, revisões, estudos qualitativos e observacionais foram excluídos.

\section{Estratégias de busca, seleção dos estudos e extração dos dados}

As estratégias de busca foram construídas de maneira peculiar para cada base de dados, utilizou-se descritores controlados e não-controlados combinados com operadores booleanos e truncamentos.

A busca e a seleção dos estudos foram realizadas em 01 de outubro de 2020, por duas revisoras (D.X.S. e L.G), de maneira independente, nas seguintes bases de dados: Pubmed/medline, PsycInfo, Web of Science e Clinical trials. Em 19 de maio de 2021 foi realizada uma atualização da busca, porém não foram encontrados novos estudos elegíveis à revisão.

Foi realizada a etapa de seleção dos estudos em duas fases, sendo a primeira, por meio da leitura de títulos e de resumos dos artigos recuperados e, a segunda, pela análise na íntegra dos estudos selecionados na etapa anterior.

A extração de dados foi realizada de maneira independente pelas revisoras citadas anteriormente. Para esta etapa utilizou-se um formulário criado pelas autoras para colher informações específicas para este estudo com informações que continham: título, autores, número de registro do ensaio clínico, ano, revista, objetivo do estudo, local de realização do estudo, tipo, intervenção, controle, número de participantes, randomização, desfechos, resultados, tipo de câncer dos participantes, fase do tratamento.

\section{Avaliação da qualidade dos estudos inclú́dos}

Utilizou-se o instrumento de avaliação do risco de viés disponibilizado pela Cochrane Collaboration, composto por sete domínios: geração da sequência de alocação, sigilo de alocação, cegamento de participantes, profissionais e avaliadores de desfecho, desfechos de dados incompletos e outras fontes de viés. Nesta ferramenta, é atribuído baixo, incerto ou alto risco de viés para cada domínio (Higgins \& Green., 2012).

\section{Resultados}

Foram recuperadas 112 referências nas seguintes bases de dados: 34 na Pubmed/Medline, 3 na PsycoInfo, 59 na Web of Science e 16 no Clinicaltrials.gov.

Após a remoção de 29 referências duplicadas, 83 títulos e resumos foram lidos e 43 foram excluídos nesta primeira etapa. Na segunda etapa, 40 publicações foram analisadas na íntegra e 24 foram excluídas. Ao final da triagem, foram incluídas na revisão 16 publicações, derivadas de 8 ensaios clínicos dos quais 6 estavam finalizados e 2 ainda em desenvolvimento. $\mathrm{O}$ processo de seleção e triagem dos estudos encontra-se descrito no fluxograma da Figura 1, a qual apresenta o detalhamento do 
processo de inclusão e exclusão dos artigos.

Figura 1 - Fluxograma de seleção dos estudos.

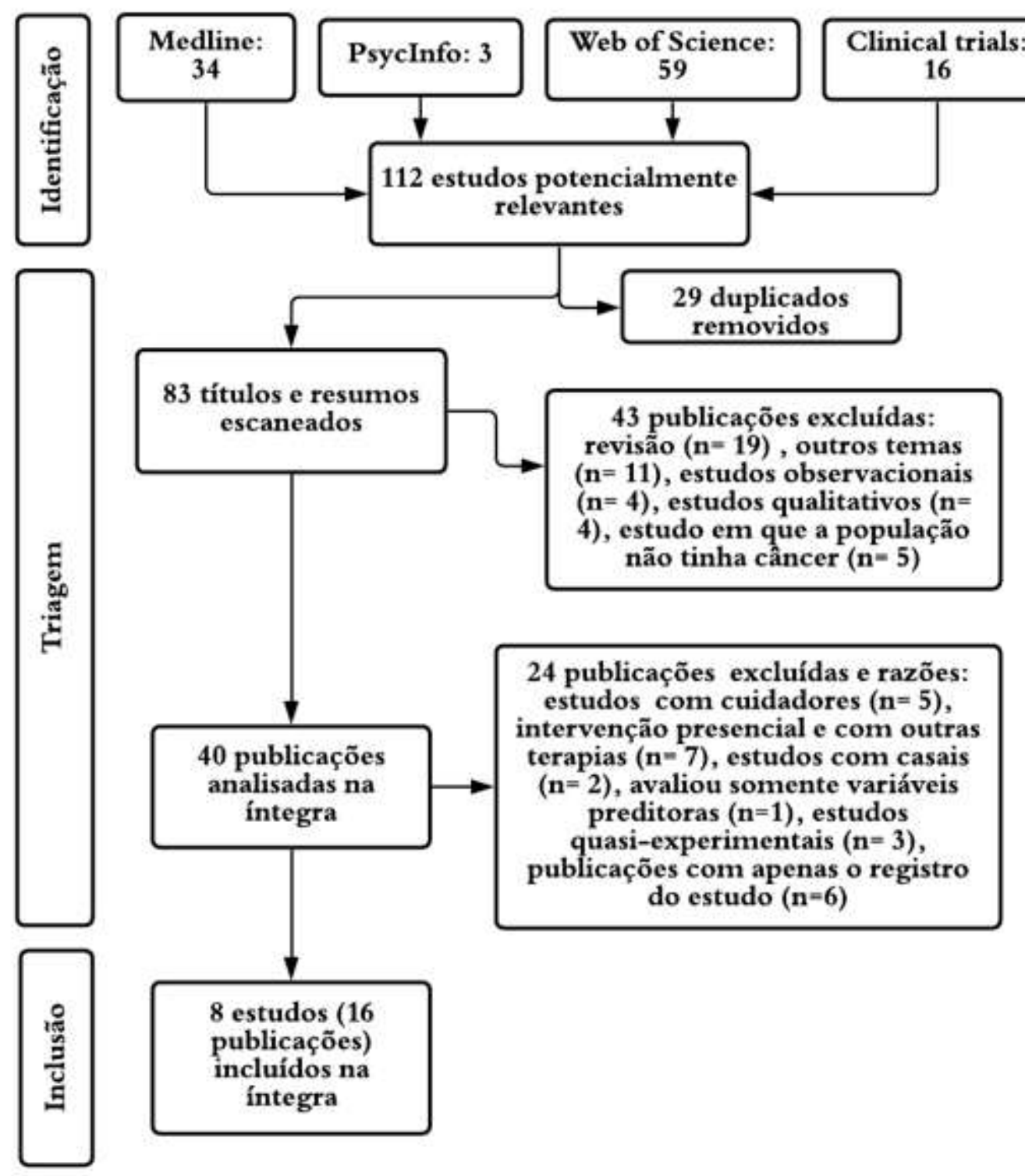

Fonte: Autores.

Os estudos incluídos nesta revisão tiveram um total de 714 participantes, com idade média de 54,69, sendo 568 participantes do sexo feminino e 146 do sexo masculino. Em relação aos diagnósticos dos participantes incluídos, dois estudos incluíram pessoas com câncer de mama e de próstata, um estudo obteve sua amostra de pessoas com diagnóstico de melanoma e os outros cinco estudos incluíram pessoas com quaisquer tipos de câncer, tais como câncer de tireóide, bexiga, cólon, colorretal, ginecológico, linfoma não Hodgkin's, câncer do sistema linfático, tumor neuroendócrino e outros tipos não detalhados nos ensaios clínicos. A amostra da pesquisa foi obtida, em sua maioria, com pessoas que haviam encerrado o tratamento para o câncer; apenas uma pesquisa foi direcionada aos pacientes na fase do tratamento quimioterápico.

As intervenções que tiveram seus efeitos analisados foram programas baseados em mindfulness implementados em formato síncrono, por meio de sessões online ou presencial, ou assíncrono por meio de website ou aplicativos. Os estudos incluídos tiveram intervenções com duração de seis a doze semanas, com sessões de tempo variável. 
Como condições controles foram utilizadas lista de espera, cuidados usuais e recebimento de e-mails psicoeducativos. Os estudos apresentaram heterogeneidade em relação à população estudada, número e duração das sessões de mindfulness, comparadores utilizados, desfechos avaliados e testes estatísticos aplicados.

As principais características dos estudos incluídos estão descritas na Tabela 1.

Tabela 1 - Características dos ensaios clínicos incluídos na revisão integrativa.

\begin{tabular}{|c|c|c|c|c|c|}
\hline $\begin{array}{l}1^{\circ} \text { autor, } \\
\text { registro clínico } \\
\text { e país }\end{array}$ & $\begin{array}{l}\text { População/cara } \\
\text { cterísticas (n) }\end{array}$ & Intervenção (n) & $\begin{array}{l}\text { Modo de } \\
\text { entrega }\end{array}$ & $\begin{array}{l}\text { Número de } \\
\text { sessões/ } \\
\text { duração }\end{array}$ & Controle (n) \\
\hline $\begin{array}{l}\text { Carlson et al. } \\
\text { 2019; } \\
\text { NCT03484000 } \\
\text { Canadá }\end{array}$ & $\begin{array}{l}\text { Adultos que } \\
\text { serão submetidos } \\
\text { à quimioterapia } \\
(\mathrm{n}=178) \text { para } \\
\text { tratamento de: } \\
\text { 1. Carcinoma de } \\
\text { mama HER } 2^{1} \\
\text { estágios I-III } \\
\text { 2.Carcinoma } \\
\text { colorretal em } \\
\text { estágio II-III }\end{array}$ & $\begin{array}{l}\text { Online } \\
\text { Mindfulness } \\
\text { Based Cancer } \\
\text { Recovery } \\
(\mathrm{MBCR})(\mathrm{n}=119)\end{array}$ & $\begin{array}{l}\text { Sessões online } \\
\text { síncronas em } \\
\text { grupo, } \\
\text { conduzidas por } \\
\text { um instrutor }\end{array}$ & $\begin{array}{l}12 \text { sessões } \\
\text { semanais/ } 55 \\
\text { minutos }\end{array}$ & $\begin{array}{l}\text { Lista de espera } \\
(\mathrm{n}=59)\end{array}$ \\
\hline $\begin{array}{l}\text { Subnis et al. } \\
2020 ; \\
\text { NCT03484000 } \\
\text { Canadá }\end{array}$ & $\begin{array}{l}\text { Adultos que } \\
\text { concluíram } \\
\text { tratamento para } \\
\text { câncer }(n=74)\end{array}$ & $\begin{array}{l}\text { Am Mindfulness- } \\
\text { Based Cancer } \\
\text { Survivorship - } \\
\text { Journey (MBCS) } \\
(\mathrm{n}=37)\end{array}$ & Aplicativo & $\begin{array}{l}4 \text { semanas/ } 20 \text { a } \\
30 \text { minutos todos } \\
\text { os dias, ou no } \\
\text { mínimo } 4 \text { dias } \\
\text { por semana }\end{array}$ & $\begin{array}{l}\text { Cuidados usuais } \\
\text { (lista de espera) } \\
(n=37)\end{array}$ \\
\hline $\begin{array}{l}\text { Russell et al. } \\
2018 ; \\
\text { Russell et al. } \\
2018 \\
\text { ACTRN126170 } \\
\text { 00081314 } \\
\text { Austrália } 2\end{array}$ & $\begin{array}{l}\text { Adultos } \\
\text { diagnosticados } \\
\text { com melanoma } \\
\text { nos estágios } 2 \mathrm{c}, \\
3 \text { a, } 3 \mathrm{~b} \text { ou } 3 \mathrm{c} \text { que } \\
\text { tenham } \\
\text { encerrado o } \\
\text { tratamento } \\
(\mathrm{n}=69)\end{array}$ & $\begin{array}{l}\text { Programa online } \\
\text { em grupo de } \\
\text { mindfulness } \\
(\mathrm{n}=46)\end{array}$ & Website & $\begin{array}{l}6 \text { semanas/ } \\
\text { duração não } \\
\text { informada }\end{array}$ & $\begin{array}{l}\text { Cuidados usuais } \\
\text { (lista de espera) } \\
(n=23)\end{array}$ \\
\hline $\begin{array}{l}\text { Compen et al } \\
2015 \\
\text { Compen et al } \\
2018 \\
\text { Cillessen et al } \\
2018 \\
\text { Compen et al } \\
2019 \\
\text { Cillessen et al } \\
2020 \\
\text { NCT02138513 } \\
\text { Holanda }\end{array}$ & $\begin{array}{l}\text { Adultos com } \\
\text { qualquer tipo ou } \\
\text { estágio de câncer } \\
(n=245)\end{array}$ & $\begin{array}{l}\text { 1: Mindfulness- } \\
\text { Based Cognitive } \\
\text { Therapy } \\
\text { (MBCT) }(\mathrm{n}=77) \\
\text { 2- Internet-based } \\
\text { Mindfulness } \\
\text { Based Cognitive } \\
\text { Therapy } \\
\text { (eMBCT) }(\mathrm{n}=90)\end{array}$ & $\begin{array}{l}\text { 1-Sessões } \\
\text { presenciais em } \\
\text { grupo, } \\
\text { conduzidas por } \\
\text { um instrutor } \\
\text { 2- Sessões } \\
\text { online síncronas } \\
\text { em grupo, } \\
\text { conduzidas por } \\
\text { um instrutor }\end{array}$ & $\begin{array}{l}8 \text { semanais/ } 2,5 \\
\text { horas }\end{array}$ & $\begin{array}{l}\text { Cuidados usuais } \\
(\mathrm{n}=78)\end{array}$ \\
\hline
\end{tabular}




\begin{tabular}{|c|c|c|c|c|c|}
\hline $\begin{array}{l}\text { Zernicke et al } \\
2013 \\
\text { Zernicke at al } \\
2014 \\
\text { Zernicke et al } \\
2016 ; \\
\text { NCT01476891 } \\
\text { Canadá }\end{array}$ & $\begin{array}{l}\text { Adultos com } \\
\text { qualquer tipo ou } \\
\text { estágio de câncer } \\
\text { que tenham } \\
\text { concluído } \\
\text { tratamento } \\
\text { primário nos } \\
\text { últimos } 36 \text { meses } \\
(n=62)\end{array}$ & $\begin{array}{l}\text { Online } \\
\text { Mindfulness- } \\
\text { Based Cancer } \\
\text { Recovery } \\
(\mathrm{MBCR})(\mathrm{n}=30)\end{array}$ & $\begin{array}{l}\text { Sessões online } \\
\text { síncronas em } \\
\text { grupo, } \\
\text { conduzidas por } \\
\text { um instrutor }\end{array}$ & $\begin{array}{l}8 \text { semanas/ } 2 \\
\text { horas, incluindo } \\
\text { retiro online de } 6 \\
\text { horas }\end{array}$ & $\begin{array}{l}\text { Cuidados usuais } \\
\text { (lista de espera) } \\
(\mathrm{n}=32)\end{array}$ \\
\hline $\begin{array}{l}\text { Wolvers et al } \\
2015 \\
\text { Bruggeman - } \\
\text { Everts et al } \\
2017 \text {; } \\
\text { NTR3483 } \\
\text { (NTR3483) } \\
\text { Holanda }\end{array}$ & $\begin{array}{l}\text { Adultos que } \\
\text { concluíram } \\
\text { tratamento para } \\
\text { câncer no } \\
\text { mínimo } 3 \text { meses } \\
\text { antes }(n=167)\end{array}$ & $\begin{array}{l}\text { 1. Web-based } \\
\text { mindfulness- } \\
\text { based cognitive } \\
\text { therapy } \\
\text { (eMBCT) }(\mathrm{n}=55) \\
\text { 2. Ambulant } \\
\text { Activity } \\
\text { Feedback (AAF) } \\
(\mathrm{n}=62)\end{array}$ & $\begin{array}{l}\text { Braço 1: Website } \\
\text { Braço 2: } \\
\text { Aplicativo e } \\
\text { acelerômetro que } \\
\text { se comunicam } \\
\text { por bluetooth }\end{array}$ & $\begin{array}{l}9 \text { semanas/ } 4 \\
\text { horas semanais }\end{array}$ & $\begin{array}{l}\text { Controle ativo: } \\
\text { recebimento de } \\
\text { e-mails } \\
\text { psicoeducativos } \\
(\mathrm{n}=50)\end{array}$ \\
\hline $\begin{array}{l}\text { Messer et al } \\
2019^{3}\end{array}$ & $\begin{array}{l}\text { Adultos } \\
\text { sobreviventes de } \\
\text { câncer, estágio } \\
\text { de I a III }(n=21)\end{array}$ & $\begin{array}{l}\text { Treinamento em } \\
\text { mindfulness } \\
(\mathrm{n}=11)\end{array}$ & Website & $\begin{array}{l}6 \text { semanas/ } \\
\text { duração não } \\
\text { informada }\end{array}$ & $\begin{array}{l}\text { Cuidados usuais } \\
(\mathrm{n}=10)\end{array}$ \\
\hline $\begin{array}{l}\text { Nissen et al } \\
2019 \\
\text { NCT03100981 } \\
\text { Suécia }\end{array}$ & $\begin{array}{l}\text { Adultos } \\
\text { sobreviventes de } \\
\text { câncer de mama } \\
(n=137) \text { e } \\
\text { próstata }(n=13)\end{array}$ & $\begin{array}{l}\text { Internet- } \\
\text { delivered } \\
\text { Mindfulness- } \\
\text { Based Cognitive } \\
\text { Therapy } \\
\text { (iMBCT }(\mathrm{n}=104)\end{array}$ & $\begin{array}{l}\text { Website, } \\
\text { contatos } \\
\text { telefônicos e } \\
\text { feedbacks por } \\
\text { escrito (enviados } \\
\text { semanalmente } \\
\text { por um } \\
\text { terapeuta) }\end{array}$ & $\begin{array}{l}8 \text { semanas/ } \\
\text { duração não } \\
\text { informada }\end{array}$ & $\begin{array}{l}\text { Lista de espera } \\
(n=46)\end{array}$ \\
\hline
\end{tabular}

Notas: 1. Tumores de mama com níveis mais altos da proteína HER2. 2. Estudo piloto. 3. Não encontramos o registro clínico desta pesquisa. Fonte: Autores.

\section{Efeitos da intervenção}

Os principais desfechos avaliados nos estudos foram: viabilidade e aceitação do programa online de mindfulness (Russell et al., 2018; Zernicke et al., 2014), sofrimento psicológico, medo de recorrência do câncer, qualidade de vida, mindfulness, custo-efetividade, saúde mental positiva (Cillessen et al., 2018; Cillessen et al., 2020; Compen et al., 2018; Compen et al., 2019), distúrbios de humor, estresse, espiritualidade (Zernicke et al., 2014; Zernicke et al., 2016), fadiga (Bruggeman -Everts et al., 2017; Messer et al., 2019), ansiedade, depressão, distúrbios do sono (Messer et al., 2019) e bemestar (Nissen et al., 2019).

Programas cujas intervenções foram oferecidas de maneira síncrona, com sessões online em grupo, conduzidas por um instrutor, foram: Carlson et al (2019), Compen et al. (2018) e Zernicke et al. (2014), sendo que Compen et al (2018) empregou também outra intervenção ativa, que foram sessões presenciais em grupo conduzidas por um instrutor. Intervenções empregadas de maneira assíncrona foram: Subnis et al (2020) por meio de um aplicativo e Russell et al (2018); Messer et al. (2019) e Nissen et al. (2019) por website; Bruggeman -Everts et al., 2017 ofereceu duas intervenções de entrega assíncrona: por meio de aplicativo e website.

Bruggeman -Everts et al (2017) constatou que o programa online de mindfulness foi mais efetivo contra a severidade da fadiga em pessoas com câncer do que a condição de controle ativo, composta por recebimento de e-mails psicoeducativos. Porém, o programa online de mindfulness foi inferior à terapia guiada por fisioterapeuta com o uso de um acelerômetro na redução da fadiga. 
Compen et al (2018) constatou que tanto a intervenção online com mindfulness como a presencial reduziram de maneira satisfatória o sofrimento psicológico em uma amostra heterogênea de pacientes com câncer, quando comparadas aos cuidados usuais.

Compen et al (2019) demonstrou que em relação ao custo-efetividade, a intervenção online e presencial com mindfulness mostrou-se superior aos cuidados usuais. Após 9 meses de follow-up houve aumento da qualidade de vida em eMBCT e MBCT. Ambas as intervenções produziram economia de custos e, simultaneamente, aumentaram a qualidade de vida dos pacientes com câncer.

Cillessen et al (2018) constatou que tanto eMBCT quanto MBCT contribuíram para mudanças importantes em desfechos relacionados a sofrimento psicológico, no entanto, observaram que pacientes com maior nível de estresse na linha de base e pacientes com nível menor de mindfulness se beneficiaram mais com o programa online de mindfulness.

Em relação à taxa de adesão ao programa online de mindfulness, Cillessen et al (2020) demonstrou que está diretamente relacionada à melhora nos desfechos avaliados.

Zernicke et al (2014) e Zernicke et al (2016) encontraram adesão e satisfação dos participantes com o programa online de mindfulness e melhoras nos desfechos de sintomas de estresse, distúrbios de humor, espiritualidade, nível de mindfulness e crescimento pós-traumático, quando comparado com cuidados usuais.

Efeitos benéficos significativos foram obtidos em todos os desfechos analisados por Messer et al (2019) após a intervenção (ansiedade, depressão, humor, qualidade do sono e fadiga), uma vez que foi observada superioridade da intervenção com mindfulness em relação aos cuidados usuais. Os pesquisadores concluem que o programa online de mindfulness é uma intervenção eficaz e acessível, que contribui para a redução do sofrimento psicológico em sobreviventes de câncer.

Nissen et al (2019) demonstrou superioridade da intervenção online com mindfulness em relação à lista de espera, uma vez que reduziu a ansiedade e sintomas depressivos após a intervenção. Os efeitos se mantiveram no período de follow-up para a ansiedade.

Os resultados de Carlson et al (2019) e Subnis et al (2020) ainda não haviam sido publicados por ocasião da revisão, mas esses autores esperam que, com o desenvolvimento de seus ensaios clínicos, haja mudanças após a intervenção com mindfulness, em desfechos relevantes para pacientes com diagnóstico de câncer, tais como: fadiga, dor, distúrbios do sono, diminuição no estresse, ansiedade, depressão, medo de recorrência do câncer, melhora no humor e qualidade de vida.

Todos os resultados dos desfechos discorridos nesta seção são apresentados detalhadamente na Tabela 2.

Tabela 2- Desfechos estudados e resultados alcançados dos estudos incluídos na presente revisão segundo autoria e ano.

\begin{tabular}{|c|c|c|}
\hline Estudos & Desfechos & Resultados \\
\hline $\begin{array}{l}\text { Carlson et al } \\
2019\end{array}$ & $\begin{array}{l}\text {-Distúrbios do sono/Índice de qualidade do sono de } \\
\text { Pittsburgh } \\
\text {-Dor/Brief Pain Inventory } \\
\text {-Náusea, vômito/Osoba Nausea and Vomiting Module } \\
\text {-Humor/Profile of Mood States-Short Form } \\
\text { (POMS-SF) } \\
\text {-Estresse/Calgary Symptoms of Stress Inventory } \\
\text { (C-SOSI) } \\
\text {-QV/Functional Assessment of Cancer Therapy - } \\
\text { General (FACT-G) } \\
\text { Desfechos exploratórios: } \\
\text {-Função cognitiva/Functional Assessment of Cancer } \\
\text { Therapy - Cog (Self-Report) Sustained Attention to } \\
\text { Response Task (Objective) -(FACTCog SART) }\end{array}$ & $\begin{array}{l}\text { Espera-se que a intervenção resulte em: menos fadiga pós- } \\
\text { tratamento quimioterápico, redução nos problemas de sono, } \\
\text { menos dor, náuseas/vômitos, melhora no humor, diminuição do } \\
\text { estresse e maior qualidade de vida após MBCR, pós } \\
\text { quimioterapia e período de seguimento }{ }^{1} \text {. }\end{array}$ \\
\hline
\end{tabular}


-Contagem de glóbulos brancos/CBC (dados do prontuário médico)

-Retorno ao trabalho/Tempo de retorno ao trabalho após licença médica (taxa de retorno ao trabalho em 12 meses)

\begin{tabular}{|c|c|}
\hline $\begin{array}{l}\text { Subnis et al } \\
2020\end{array}$ & $\begin{array}{l}\text { Sintomas de estresse; } \\
\text { Medo da recorrência do câncer; } \\
\text { Ansiedade; Depressão; Fadiga; Funcionamento físico } \\
\text { geral; Mindfulness; Ruminação; Evitação experiencial; } \\
\text { Retorno ao trabalho }\end{array}$ \\
\hline $\begin{array}{l}\text { Russell } \\
\text { et al } 2018 \\
\text { Russell et al } \\
2018\end{array}$ & $\begin{array}{l}\text { - Viabilidade do programa } \\
\text { - Aceitação/ feedback sobre o conteúdo do programa } \\
\text { - Medo da recorrência do câncer/ FCRI; } \\
\text { - Preocupações/PSQW-A } \\
\text { - Ruminação/RRQ-Rum; } \\
\text { - Estresse percebido/ PSS10; } \\
\text { - Traço de mindfulness/ CAMS-R }\end{array}$ \\
\hline
\end{tabular}

Compen et al - Sofrimento psicológico/HADS;

2015 - Medo de recorrência do câncer/FCRI;

Compen et al - Ruminação/ RRQ;

2018 - Saúde mental positiva/ MHC-SF;

Cillessen et al - Transtornos de humor ou ansiedade do Eixo I, DSM-

2018

Compen et al

2019

Cillessen et al 2020
Espera-se a intervenção seja eficaz para a redução do medo de recorrência do câncer, ansiedade, depressão, fadiga e outros desfechos propostos no estudo ${ }^{1}$.

Viabilidade e aceitação da intervenção foi confirmada em uma taxa de $72 \%$ por parte dos respondentes que consideraram a intervenção proveitosa. Houve redução significativa do medo de recorrência do câncer comparado com o grupo controle (diferença das médias $=-2,55 ; 95 \%$ CI $-4.43,-0,67 ; \mathrm{p}=$ $0,008)$. Os dois grupos não diferiram na preocupação, ruminação, estresse percebido ou resultados de atenção plena.

Tanto o MBCT quanto o eMBCT resultaram em uma redução estatisticamente significativa e clinicamente confiável do sofrimento psicológico em comparação com o TAU. As intervenções também trouxeram: diminuição do medo de recorrência do câncer no eMBCT e MBCT (d de Cohen, 0,53 e $0,27$, respectivamente) e MBCT (d de Cohen $=0,27)$, redução da ruminação (d de Cohen, 0,51 e 0,42, respectivamente), aumento na qualidade de vida relacionada à saúde mental (d de Cohen, 0,67 e 0,59, respectivamente), aumento nos escores de atenção plena (d de Cohen, 0,82 e 0,47, respectivamente), aumento na saúde mental positiva em eMBCT e MBCT (d de Cohen, 0,44 e 0,12, respectivamente), aumento da qualidade de vida (eMBCT x TAU, d de Cohen=0.54; MBCT x TAU, d de Cohen= 0.53).

Houve manutenção do aumento da qualidade de vida durante o período de follow up, mas não houve diferenças com significância entre os grupos eMBCT e MBCT.

Não houve aumento da qualidade de vida relacionada à saúde física eMBCT e MBCT (d de Cohen,0,24 e 0,35, respectivamente).

Observou-se que na linha de base houve melhora mais elevada no sofrimento psicológico dos pacientes com neuroticismo maior, tanto no grupo eMBCT e MBCT, quando comparado com o TAU (eMBCT $\vee$ TAU P $=0,004 ;$ MBCT $\vee$ TAU P $=$ $0,014)$.

Com relação ao custo efetividade, constatou-se que durante o período da intervenção as perdas de produtividade e custos sociais ligadas ao trabalho remunerado foram menores tanto no eMBCT quanto no MBCT, em comparação com TAU.

Participantes que completaram todos os exercícios em MBCT relataram menos sofrimento psicológico $(\mathrm{t} 86=-2,47, \mathrm{P}=0,02)$ e mais saúde mental positiva após a intervenção do que aqueles que não responderam a todas a questões $(\mathrm{t} 86=5,18, \mathrm{P}=0,02)$. Nas análises das medidas: tempo médio e total de login, número de logins, e-mails enviados e exercícios concluídos, houve sofrimento psicológico menor ( $\mathrm{t} 86=-2.80, \mathrm{P}=.01)$ e mais saúde mental positiva ( $\mathrm{t} 86=5,24, \mathrm{P}=0,01$ ) para aqueles que concluíram todos os exercícios. 


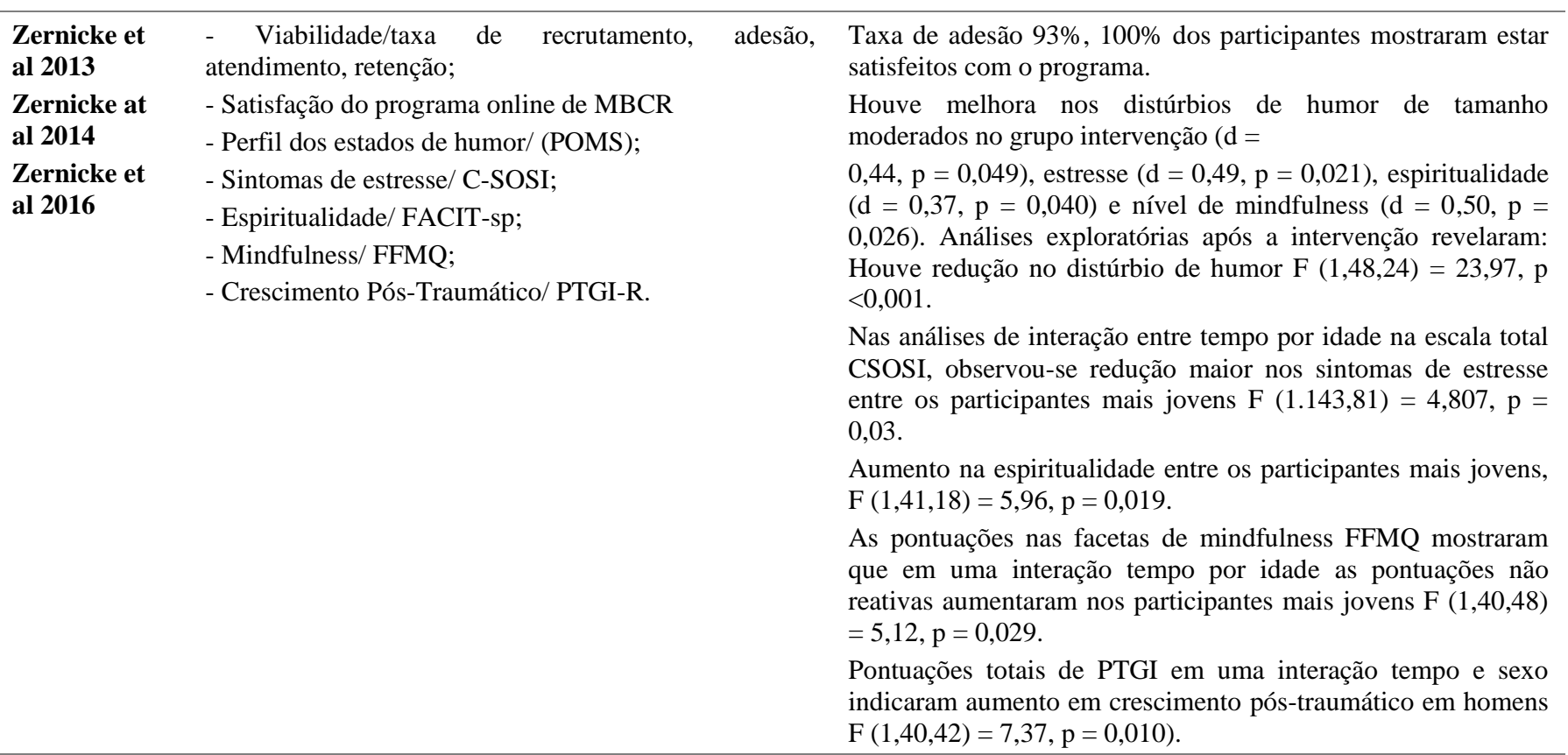

\begin{tabular}{|c|c|c|}
\hline $\begin{array}{l}\text { Wolvers et al } \\
2015\end{array}$ & $\begin{array}{l}\text { - Severidade da fadiga/ CIS-SF-Fatigue Severity } \\
\text { Subscale; }\end{array}$ & $\begin{array}{l}\text { Diminuição na severidade da fadiga em } 66 \% \text { dos participantes } \\
\text { no grupo AAF }(41 / 62), 49 \%(27 / 55) \text { naqueles no grupo }\end{array}$ \\
\hline $\begin{array}{l}\text { Bruggeman - } \\
\text { Everts et al } \\
2017\end{array}$ & $\begin{array}{l}\text { - Outras dimensões da fadiga/ CIS-Physical and } \\
\text { Cognitive Fatigue and Motivation Subscales; } \\
\text { - Afeto/ PANAS; } \\
\text { - Sofrimento psicológico/ HADS; }\end{array}$ & $\begin{array}{l}\text { eMBCT e } 12 \% \text { no grupo psicoeducação. } \\
\text { Nesta publicação avaliou-se apenas a fadiga; os autores } \\
\text { registraram que os demais desfechos serão avaliados em } \\
\text { publicações posteriores. }\end{array}$ \\
\hline
\end{tabular}

- Capacidade autopercebida para trabalho/ WAS;

- Retorno ao trabalho e horas de trabalho/ questões adaptadas do TiC-P;

- Mindfulness/ FMI;

- Atividade física/ Accelerometer ProMove 3D;

- Qualidade do sono/ Subjective Sleep Quality Scale;

- Sensação de controle sobre a fadiga/ Self-Efficacy Scale;

Credibilidade e expectativa/ CEQ;

- Aliança de trabalho/WAI-SF;

- Autoeficácia sobre atividade física/ Quatro questões de autoconceitualização sobre a percepção do volume de atividades, comparação do volume, satisfação com volume de atividades;

- Catastrofização da fadiga/ FCS;

- Medo de recorrência do câncer/ 2 itens selecionados de uma escala Likert;

- Atribuições causais/ Pergunta de resposta aberta: "O que você considera como a causa do seu cansaço?"

$\begin{array}{ll}\text { Messer et al } & \text { - Ansiedade e depressão/ HAS; } \\ & \text { - Humor/ POMS-SF; } \\ & \text { - Qualidade do sono/ PSQI; } \\ & \text { - Fadiga/ FSI. }\end{array}$

A intervenção trouxe redução nas seguintes medidas: ansiedade e depressão beneficiadas (HADS F $(1,19)=7,33$,

$\mathrm{p}=0,014$, gav $=0,64)$, distúrbios no sono (PSQI F $(1,19)=$ $12,44, \mathrm{p}=0,0023$, gav $=1,14)$ e fadiga (FSI $\mathrm{F}(1,19)=5,96, \mathrm{p}$ $=0,025$, gav $=1,03)$.

$\begin{array}{lll}\begin{array}{l}\text { Nissen et al } \\ \mathbf{2 0 1 9}\end{array} & \text { - Ansiedade e depressão/ Subescala de estado do } & \text { Com a intervenção houve melhora na ansiedade }(\mathrm{d} \text { de Cohen }= \\ & \text { STAI-Y e o BDI-II; } & 0,45 ; \mathrm{P}=0,017) \text { e nos sintomas depressivos }(\mathrm{d}=0,42 ; \mathrm{P}= \\ & \text { - Estresse/ PSS10; } & 0,024) \text { Efeitos significativos entre os grupos foram } \\ & \text { - Bem-estar/ Bem-estar/WHO-5; } & \text { encontrados para o bem-estar na avaliação de acompanhamento } \\ & \text { - Distúrbio do sono/ ISI. } & \text { de } 6 \text { meses }(\mathrm{d}=0,48) \text {. Somente os efeitos para ansiedade } \\ & \text { mantiveram-se no período de follow-up. Não foi alcançada } \\ & \text { significância estatística para bem-estar, percepção do estresse e } \\ \text { insônia. }\end{array}$


Notas: FCRI= Fear of Cancer Recurrence Inventory; PSQW-A= Penn State Worry Questionnaire-Abbreviated; RRQ-Rum= Reflection Questionnaire-rumination subscale; PSS10= Perceived Stress Scale; CAMS-R: Cognitive and Affective Mindfulness Scale-Revised; HADS= Hospital Anxiety and Depression-Scale; FCRI= Fear of Cancer Recurrence ; RRQ= Rumination Reflection Questionnaire; MHC-SF= Mental Health Continuum - Short Form; SCID= Structural Clinical Interview for DSM-IV Axis I Disorders; NEO-FFI= Personality Dimensions; FFMQ$\mathrm{SF}=$ Five Facet Mindfulness Questionary - Short Form; MAAS= Mindful Attention Awareness Scale; WAI= Working Alliance Inventory; GCQ= Group Cohesion; I-PANAS-SF= International Positive and Negative Affect Scale - Short Form; TIC-P= Health Care Costs and Productivity; EQ5D Health-Related Quality of Life (Preference-Based); SF-12 Health-Related Quality of Life (General Health Profile); MBCR= MindfulnessBased Cancer Recovery; POMS= Profile of Mood States; C-SOSI= Calgary Symptoms of Stress Inventory; FACIT-sp= Functional Assessment of Chronic Illness Therapy-Spiritual Well-Being; PTGI-R= Post-Traumatic Growth Inventory-Revised; CIS-SF = Checklist for Individual Strength Fatigue Severity Subscale; PANAS = Positive and Negative Affect Scale; WAS= Work Ability Score; TiC-P= Psychiatric IIIness; FMI= Freiburg Mindfulness Inventory; WAI-SF= Working Alliance Inventory - Short For; CEQ= Credibility and Expectancy Questionnaire; FCS= Fatigue Catastrophizing Scale; POMS-SF= Profile of Mood States-Short Form; PSQI= Pittsburgh Sleep Quality Index; FSI= Fatigue Symptom Inventory; STAI-Y= State-Trait Anxiety Inventory Y-Form; BDI= Beck Depression Inventory; WHO-5= World Health Organization Well-Being Index; ISI= Insomnia Severity Index. Gav= magnitude do efeito. Fonte: Autores.

\section{Avaliação do risco de viés dos estudos incluídos}

Em relação à geração da sequência de alocação, todos os estudos incluídos nesta análise tiveram baixo risco de viés para esta variável (Bruggeman-Everts et al., 2017; Compen et al., 2018; Messer et al., 2019; Nissen et al., 2019; Russell et al., 2018; Zernicke et al., 2014).

Na variável sigilo de alocação, somente um estudo obteve risco de viés incerto (Nissen et al., 2019), os demais obtiveram baixo risco de viés (Bruggeman-Everts et al., 2017; Compen et al., 2018; Messer et al., 2019; Russell et al., 2018; Zernicke et al., 2014).

Em relação ao cegamento de participantes e pessoal, quatro estudos apresentaram baixo risco de viés para este domínio (Compen et al., 2018; Messer et al., 2019; Russell et al., 2018; Zernicke et al., 2014), um estudo foi classificado com alto risco de viés (Bruggeman-Everts et al., 2017) e outro com risco incerto (Nissen et al., 2019).

Quanto ao cegamento dos avaliadores de desfecho, Compen et al. (2018) e Russell et al. (2018) obtiveram risco incerto de viés, três estudos tiveram baixo risco de viés (Bruggeman-Everts et al., 2017; Messer et al., 2019; Zernicke et al., 2014) e um estudo apresentou alto risco de viés (Nissen et al., 2019).

Em relação ao uso da análise por intenção de tratar, cinco estudos apresentaram baixo risco de viés para este domínio (Russell et al., 2018; Compen et al., 2018; Zernicke et al., 2014; Bruggeman-Everts et al., 2017; Nissen et al., 2019) e apenas Messer et al. (2019) apresentou alto risco de viés.

No domínio relacionado ao relato de todos os desfechos propostos nos estudos, apenas dois estudos apresentaram alto risco de viés (Bruggeman-Everts et al., 2017; Nissen et al., 2019) e quatro estudos obtiveram baixo risco de viés (Compen et al., 2018; Messer et al., 2019; Russell et al., 2018; Zernicke et al., 2014).

A avaliação do risco de viés dos estudos está sumarizada na Tabela 3.

Tabela 3- Avaliação do risco de viés.

\begin{tabular}{lllllll}
$\begin{array}{l}\text { Estudos } \\
\text { Registro clínico }\end{array}$ & $\begin{array}{l}\text { Randomizaçã } \\
\text { o }\end{array}$ & $\begin{array}{l}\text { Sigilo de } \\
\text { alocação }\end{array}$ & $\begin{array}{l}\text { Cegamento dos } \\
\text { participantes e } \\
\text { equipe }\end{array}$ & $\begin{array}{l}\text { Cegamento dos } \\
\text { avaliadores de } \\
\text { desfecho }\end{array}$ & $\begin{array}{l}\text { Dados } \\
\text { completos de } \\
\text { desfecho ou } \\
\text { análise por } \\
\text { intenção de } \\
\text { tratar }\end{array}$ & $\begin{array}{l}\text { Todos os } \\
\text { desfechos } \\
\text { foram } \\
\text { relatados }\end{array}$ \\
\hline $\begin{array}{l}\text { Russell } \\
\text { et al 2018 } \\
\begin{array}{l}\text { ACTRN1261700 } \\
\text { o081314 }\end{array}\end{array}$ & Baixo & Baixo & Baixo & Incerto & Baixo & Baixo \\
\hline
\end{tabular}




\begin{tabular}{|c|c|c|c|c|c|c|}
\hline $\begin{array}{l}\text { Compen et al } \\
2015 \\
\text { Compen et al } \\
2018 \\
\text { Cillessen et al } \\
2018 \\
\text { Compen et al } \\
2019 \\
\text { Cillessen et al } \\
2020 \\
\text { NCT02138513 }\end{array}$ & Baixo & Baixo & Baixo & Incerto & Baixo & Baixo \\
\hline $\begin{array}{l}\text { Zernicke et al } \\
2013 \\
\text { Zernicke at al } \\
2014 \\
\text { Zernicke et al } \\
2016 \\
\text { NCT01476891 }\end{array}$ & Baixo & Baixo & Baixo & Baixo & Baixo & Baixo \\
\hline $\begin{array}{l}\text { Wolvers et al } \\
2015 \\
\text { Bruggeman - } \\
\text { Everts et al } 2017 \\
\text { NTR3483 }\end{array}$ & Baixo & Baixo & Alto & Alto & Alto & Alto \\
\hline $\begin{array}{l}\text { Messer et al } \\
2019^{1}\end{array}$ & Baixo & Baixo & Baixo & Baixo & Alto & Baixo \\
\hline $\begin{array}{l}\text { Nissen et al } 2019 \\
\text { NCT03100981 }\end{array}$ & Baixo & Incerto & Incerto & Alto & Baixo & Alto \\
\hline
\end{tabular}

Nota: 1. Não encontramos o registro clínico desta pesquisa. Fonte: Autores.

\section{Discussão}

Esta revisão analisou ECRs que tiveram como objetivo avaliar os efeitos de intervenções baseadas em mindfulness desenvolvidas por meio da internet para pacientes com diagnóstico de câncer.

As intervenções online baseadas em mindfulness avaliadas nos estudos incluídos, evidenciaram efeitos positivos em desfechos relevantes para pacientes com diagnóstico de câncer, tais como: viabilidade do programa online de mindfulness, ansiedade, depressão, medo de recorrência do câncer, estresse, espiritualidade, mindfulness, distúrbios do sono, fadiga, crescimento pós-traumático, bem-estar.

Verificou-se que em Russell et al 2018, a viabilidade da intervenção foi confirmada, uma vez que $72 \%$ dos participantes consideraram a intervenção proveitosa. No estudo de Zernicke et al 2014, a taxa de adesão à intervenção foi de 93\%, e 100\% dos participantes declararam estar satisfeitos com o programa. Corroborando com esses achados, ECR realizado com participantes portadores de esclerose múltipla, em que a intervenção foi um programa online baseado em mindfulness demonstrou ser viável e aceitável, uma vez que $87 \%$ dos participantes completaram as oito semanas do programa online (Dunne et al., 2020). Outro estudo com pacientes com Parkinson, em que foi ministrada intervenção com mindfulness via internet, constatou que 73,3\% dos participantes aderiram a quatro ou mais das oito sessões o que demonstrou a viabilidade da pesquisa (Bogosian et al., 2021).

Além da viabilidade do programa online, os estudos (Compen et al., 2015; Russell et al., 2018; Zernicke et al., 2014), avaliaram outros desfechos relevantes e constataram que o medo de recorrência do câncer foi reduzido de maneira significativa. Um ECR com 84 mulheres sobreviventes de câncer de mama comparou MBSR a cuidados usuais e obteve resultados significativos na redução do medo de recorrência (Lengacher et al., 2009). Outro ECR com 322 mulheres que 
tiveram câncer de mama, obteve melhora prolongada em relação ao medo de recorrência geral para o grupo MBSR (BC), quando comparado com o tratamento usual (Lengacher et al., 2016). Medo de recorrência já foi apontado como um dos problemas mais comuns relatados por sobreviventes de câncer (Tauber et al., 2019; Simard et al., 2013) e os resultados das MBIs em relação a este sintoma têm se mostrado promissores (Ahmadiqaragezlou et al., 2020).

Considerando desfechos relativos à saúde mental, transtornos de humor, ansiedade e sofrimento psicológico avaliados apresentaram melhora moderada em (Zernicke et al., 2014), redução de sintomas em (Messer et al., 2019; Nissen et al., 2019), e os efeitos para ansiedade mantiveram-se no período de seguimento em (Nissen et al., 2019). Compen et al (2015) obteve redução estatisticamente significativa e clinicamente confiável do sofrimento psicológico tanto por MBI presencial como online, em comparação aos cuidados usuais. Já em (Russell et al., 2018) não foram encontradas diferenças na preocupação, ruminação e estresse percebido, e em (Nissen et al., 2019) não foi alcançada significância estatística para percepção do estresse. Análises de interação tempo por idade trouxeram em (Zernicke et al., 2014) redução nos níveis de estresse entre os mais jovens.

Diversos estudos na literatura corroboram com vários desses achados e podem ser sincronizados com a presente revisão. Um ensaio clínico com breve programa online de mindfulness, em que os participantes foram funcionários e estudantes, demonstrou melhora significativa em desfechos como habilidades de mindfulness, estresse percebido, ansiedade e depressão com tamanhos de efeitos de pequeno a médio (Cavanagh et al., 2018).

Uma revisão sistemática que envolveu 1571 participantes na metanálise, avaliou os efeitos da aplicação do programa de mindfulness baseado na redução do estresse (MBSR) a mulheres diagnosticadas com câncer de mama comparados a controles passivos. A curto prazo foi observada uma leve melhora nos níveis de ansiedade e depressão, com evidências moderadas e alta, respectivamente. Os resultados de médio prazo para ansiedade trouxeram uma pequena redução, com efeito moderado (Schell et al., 2019).

Em análises de interação tempo por idade, os resultados obtidos por (Zernicke et al., 2014) demonstraram ampliação nos níveis de espiritualidade e aumento nas facetas de mindfulness nos indivíduos mais jovens, houve ainda aumento do crescimento pós-traumático em homens.

Um ensaio clínico com população heterogênea para diversos tipos de câncer comparou os efeitos de um programa baseado em mindfulness com lista de espera nas variáveis espiritualidade e crescimento pós-traumático, verificou-se efeitos moderado e pequeno, respectivamente, no grupo da intervenção. Os pesquisadores concluíram que mindfulness estimulou o desenvolvimento do senso de propósito de vida, trouxe paz interior, conectividade e fortalecimento da fé em indivíduos com câncer (Labelle et al., 2015). Esse despertar de desfechos positivos, tais como aperfeiçoamento da espiritualidade e crescimento pós-traumático está associado aumento da qualidade de vida e diminuição da angústia e depressão em pessoas que tiveram câncer. (Carver \& Antoni, 2004; Morrill et al., 2008).

Compen et al (2015) compararam tanto um programa online de mindfulness quanto um presencial e verificaram que ambos contribuíram para o aumento nos índices de mindfulness e aumento na saúde mental positiva. Contudo, em (Russell et al., 2018) não foram encontradas diferenças nos resultados para mindfulness, nos grupos intervenção e controle. Em (Nissen et al., 2019) constatou-se efeitos significativos para o bem-estar entre os grupos no acompanhamento de seis meses.

Outro estudo controlado, direcionado a adultos mentalmente saudáveis, porém com queixa de estresse, empregou quatro condições de intervenção, a saber: grupo com mindfulness, intervenção auto-dirigida com mindfulness, grupo de discussão e grupo controle, demonstrou mudanças significativas nas habilidades de mindfulness, regulação da emoção e estresse psicológico, com tamanhos de efeito que variaram de médio a grande. Outro resultado observado foi uma maior eficácia da intervenção quando realizada em grupo (Ying et al., 2018). 
Um ensaio clínico não randomizado realizado com amostra heterogênea para pacientes com câncer, em que a intervenção foi um programa presencial de oito semanas de mindfulness (MBSR) e seis semanas de cura por meio de artes criativas (HC), demonstrou melhora mais acentuada nos níveis de ansiedade, sintomas de estresse, humor e aumento da espiritualidade no grupo de MBSR. Houve aumento significante do crescimento pós-traumático tanto no grupo MBSR quanto no HC (Garland et al., 2007).

Revisão sistemática direcionada a programas online de mindfulness e relaxamento para pacientes com problemas de saúde como câncer, síndrome do intestino irritável, dor crônica, fibromialgia, enxaqueca, hipertensão e cirurgia encontrou efeitos benéficos da intervenção no bem-estar e concluiu que esse tipo de terapêutica pode ser utilizado de maneira complementar ao tratamento convencional (Mikolasek et al., 2017).

Outra revisão sistemática desenvolvida por (Spijkerman et al., 2016) encontrou benefícios de intervenções online com mindufulness na ansiedade, depressão, habilidades de mindfulness, estresse. Em análises exploratórias, observou-se que na intervenção online guiada houve efeitos positivos mais elevados no estresse e habilidades de mindfulness em comparação à intervenção online não guiada.

A fadiga foi outro desfecho relevante analisado por dois estudos incluídos nesta revisão. (Bruggeman -Everts et al., 2017) detectaram redução na fadiga em $49 \%$ dos participantes que estavam no grupo mindfulness via internet. (Messer et al., 2019) ratifica este achado, uma vez que houve decréscimo da fadiga nos participantes da intervenção online com mindfulness. Revisão sistemática com metanálise em que os estudos incluídos aplicaram programas de mindfulness via internet, verificou que houve diminuição significativa da fadiga pós-intervenção e redução moderada no período de acompanhamento no grupo intervenção (Johns et al., 2021). Metanálise conduzida por Xie et al. (2020) afirma que um programa supervisionado de MBSR em um regime de oito semanas, uma vez por semana e com duas horas de duração pode ser importante estratégia terapêutica complementar para manejo da fadiga relacionada ao câncer, especialmente em pacientes com câncer de pulmão e mama.

É importante ressaltar outros desfechos relevantes incluídos nos estudos desta revisão: custo-efetividade, qualidade de vida e distúrbios do sono. Compen et al 2019 demonstrou que tanto a intervenção online quanto presencial de mindfulness, para pacientes com câncer, produziram economia de custos e aumento da qualidade de vida quando comparada com cuidados usuais. A análise de custo-efetividade de um ECR que teve como intervenção um programa de mindfulness na internet para 389 adultos com depressão residual, demonstrou que os custos gerais de incremento do programa foram $\$ 431,54$ em um ano. Os autores concluíram que esse tipo de programa é econômico, além de ser eficaz em reduzir depressão residual e prevenir recaídas e deve ser implementado nos serviços de saúde mental (Boggs et al., 2021).

Messer et al., (2019) demonstrou que houve redução significativa no distúrbio do sono de participantes do grupo online de mindfulness. Corroborando com este achado, uma revisão sistemática realizada com estudos em que se implementou programas virtuais com mindfulness no período da pandemia, constatou a eficácia destes em minimizar desarranjos moderados e severos em padrões de sono (Jiang et al., 2021).

Os estudos analisados na amostra desta revisão demonstraram efeitos importantes das intervenções baseadas em mindfulness na internet em variáveis psicológicas e de saúde que podem afetar a qualidade de vida de pacientes com diagnóstico de câncer.

Algumas limitações podem ser apontadas nesta revisão: apenas quatro bases de dados foram incluídas, estudos que apresentaram apenas o registro clínico não foram incluídos e a literatura cinzenta não foi consultada. A maioria dos estudos incluídos na análise foi constituída por uma amostra pequena, além de serem heterogêneos quanto à população, modo de entrega da intervenção, duração das sessões e desfechos avaliados, fatores que dificultaram a sumarização dos resultados. 


\section{Conclusão}

Intervenções baseadas em mindfulness por meio da internet tem efeitos benéficos para pacientes com diagnóstico de câncer e tem se mostrado terapias promissoras para a promoção da saúde com foco na qualidade de vida e redução do sofrimento psicológico.

Futuros ensaios clínicos bem delineados, com amostras robustas e que comparem MBIs online e presenciais, de maneira síncrona e assíncrona, direcionados à população com câncer, poderão contribuir significativamente para o desenvolvimento de um corpo de evidências sólidos nesta temática. A investigação da eficácia de programas de mindfulness mais curtos direcionados a pessoas com câncer também seria oportuna, considerando a adaptação às diferentes condições de saúde e fases de tratamento.

\section{Referências}

Boggs, J. M., Ritzwoller, D. P., Beck, A., Dimidjian, S., \& Segal, Z. V. (2021). Cost-Effectiveness of a web-based program for residual depressive symptoms: Mindful mood balance. Psychiatric Services (Washington, D.C.), appips202000419. Advance online publication. https://doi.org/10.1176/appi.ps.202000419

Bogosian, A., Hurt, C. S., Hindle, J. V., McCracken, L. M., Vasconcelos e Sa, D. A., Axell, S., Tapper, K., Stevens, J., Hirani, P. S., Salhab, M., Ye, W., \& Cubi-Molla, P. (2021). Acceptability and feasibility of a mindfulness intervention delivered via videoconferencing for people with Parkinson's. Journal of Geriatric Psychiatry and Neurology. 0891988720988901. https://doi.org/10.1177/0891988720988901

Bruggeman-Everts, F. Z., Wolvers, M., van de Schoot, R., Vollenbroek-Hutten, M., \& Van der Lee, M. L. (2017). Effectiveness of two web-based interventions for chronic cancer-related fatigue compared to an cctive control condition: Results of the "Fitter na kanker" randomized controlled trial. Journal of Medical Internet Research, 19(10), e336. https://doi.org/10.2196/jmir.7180

Carlson, L. E., Toivonen, K., \& Subnis, U. (2019). Integrative approaches to stress management. The Cancer Journal, 25(5), 329-336. doi: 10.1097/PPO.0000000000000395

Carlson, L. E., Subnis, U. B., Piedalue, K. A. L., Vallerand, J., Speca, M., Lupichuk, S., ... \& Wolever, R. Q. (2019). The ONE-MIND Study: Rationale and protocol for assessing the effects of ONlinE MINDfulness-based cancer recovery for the prevention of fatigue and other common side effects during chemotherapy. European Journal of Cancer Care, 28(4), e13074. https://doi.org/10.1111/ecc.13074

Carver, C. S., \& Antoni, M. H. (2004). Finding benefit in breast cancer during the year after diagnosis predicts better adjustment 5 to 8 years after diagnosis. Health Psychology: Official Journal of the Division of Health Psychology, American Psychological Association, 23(6), 595-598. https://doi.org/10.1037/02786133.23.6.595

Cavanagh, K., Churchard, A., O'Hanlon, P., Mundy, T., Votolato, P., Jones, F., Gu, J., \& Strauss, C. (2018). A randomised controlled trial of a brief online mindfulness-based intervention in a non-clinical population: Replication and extension. Mindfulness, 9(4), 1191-1205. https://doi.org/10.1007/s12671-0170856-1

Cillessen, L., Schellekens, M., Van de Ven, M., Donders, A., Compen, F. R., Bisseling, E. M., Van der Lee, M. L., \& Speckens, A. (2018). Consolidation and prediction of long-term treatment effect of group and online mindfulness-based cognitive therapy for distressed cancer patients. Acta Oncologica (Stockholm, Sweden), 57(10), 1293-1302. https://doi.org/10.1080/0284186X.2018.1479071

Cillessen, L., Johannsen, M., Speckens, A., \& Zachariae, R. (2019). Mindfulness-based interventions for psychological and physical health outcomes in cancer patients and survivors: A systematic review and meta-analysis of randomized controlled trials. Psycho-Oncology, 28(12), 2257-2269. https://doi.org/10.1002/pon.5214

Cillessen, L., van de Ven, M. O., Compen, F. R., Bisseling, E. M., van der Lee, M. L., \& Speckens, A. E. (2020). Predictors and effects of usage of an online mindfulness intervention for distressed cancer patients: Usability study. Journal of Medical Internet Research, 22(10), e17526. https://doi.org/10.2196/17526

Compen, F. R., Bisseling, E. M., Van der Lee, M. L., Adang, E. M., Donders, A. R., \& Speckens, A. E. (2015). Study protocol of a multicenter randomized controlled trial comparing the effectiveness of group and individual internet-based Mindfulness-Based Cognitive Therapy with treatment as usual in reducing psychological distress in cancer patients: the BeMind study. BMC Psychology, 3(1), 27. https://doi.org/10.1186/s40359-015-0084-1

Compen, F.R., Bisseling, E., Schellekens, M., Donders, R., Carlson, L., Lee, M., Speckens, A. (2018) Face-to-face and internet-based Mindfulness-Based Cognitive Therapy compared with treatment as usual in reducing psychological distress in patients with cancer: A multicenter randomized controlled trial. Journal of Clinical Oncology, 36(23):2413-2421. DOI:10.1200/JCO.2017.76.5669

Dunne, J., Chih, H. J., Begley, A., Daly, A., Gerlach, R., Schütze, R., Castell, E., Byrne, J., \& Black, L. J. (2021). A randomised controlled trial to test the feasibility of online mindfulness programs for people with multiple sclerosis. Multiple sclerosis and related disorders, $48,102728$. https://doi.org/10.1016/j.msard.2020.102728

Gardner-Nix, J., Backman, S., Barbati, J., \& Grummitt, J. (2008). Evaluating distance education of a mindfulness-based meditation programme for chronic pain management. Journal of Telemedicine and Telecare, 14(2), 88-92. https://doi.org/10.1258/jtt.2007.070811.

Garland, S. N., Carlson, L. E., Cook, S., Lansdell, L., \& Speca, M. (2007). A non-randomized comparison of mindfulness-based stress reduction and healing arts programs for facilitating post-traumatic growth and spirituality in cancer outpatients. Supportive Care in Cancer : Official Journal of the Multinational Association of Supportive Care in Cancer, 15(8), 949-961. https://doi.org/10.1007/s00520-007-0280-5 
Higgins, J. P., Thomas, J., Chandler, J., Cumpston, M., Li, T., Page, M. J., \& Welch, V. A. (Eds.). (2019). Cochrane handbook for systematic reviews of interventions. John Wiley \& Sons.

Jiang, A., Rosario, M., Stahl, S., Gill, J. M., \& Rusch, H. L. (2021). The effect of virtual mindfulness-based interventions on sleep quality: A systematic review of randomized controlled trials. Current Psychiatry Reports, 23(9), 62. https://doi.org/10.1007/s11920-021-01272-6

Johns, S. A., Tarver, W. L., Secinti, E., Mosher, C. E., Stutz, P. V., Carnahan, J. L., Talib, T. L., Shanahan, M. L., Faidley, M. T., Kidwell, K. M., \& Rand, K. L. (2021). Effects of mindfulness-based interventions on fatigue in cancer survivors: A systematic review and meta-analysis of randomized controlled trials Critical Reviews in Oncology/Hematology, 160,103290. https://doi.org/10.1016/j.critrevonc.2021.103290

Kinner, E. M., Armer, J. S., McGregor, B. A., Duffecy, J., Leighton, S., Corden, M. E., Gauthier Mullady J., Penedo F.J., \& Lutgendorf, S. K. (2018). Internetbased group intervention for ovarian cancer survivors: Feasibility and preliminary results. JMIR Cancer, 4(1), e8430. DOI:10.2196/cancer.8430

Kubo, A., Kurtovich, E., McGinnis, M., Aghaee, S., Altschuler, A., Quesenberry Jr, C., Kolevska, T., Liu, R., Greyz-Yusupov, N., \& Avins, A. (2020). Pilot pragmatic randomized trial of mHealth mindfulness-based intervention for advanced cancer patients and their informal caregivers. Psycho-Oncology. https://doi.org/10.1002/pon.5557

Kuderer, N. M., Choueiri, T. K., Shah, D. P., Shyr, Y., Rubinstein, S. M., Rivera, D. R., Sethe, S., Hsu, C.Y, Desai, A., Lopes Jr., G.L., Grivas, P., Painter C.A., Peters, S., Thompson M.A., Bakouny, Z., Batist, G., Bekaii-Saab, T., Bilen, M.A., Bouganim, N., ... \& Loaiza-Bonilla, A. (2020). Clinical impact of COVID-19 on patients with cancer (CCC19): A cohort study. The Lancet, 395(10241), 1907-1918. https://doi.org/10.1016/S0140-6736(20)31187-9

Labelle, L. E., Lawlor-Savage, L., Campbell, T. S., Faris, P., \& Carlson, L. E. (2015). Does self-report mindfulness mediate the effect of Mindfulness-Based Stress Reduction (MBSR) on spirituality and posttraumatic growth in cancer patients? The Journal of Positive Psychology, 10(2), 153-166. DOI: $10.1080 / 17439760.2014 .927902$

Lengacher, C. A., Johnson-Mallard, V., Post-White, J., Moscoso, M. S., Jacobsen, P. B., Klein, T. W., Widen, R. H., Fitzgerald, S. G., Shelton, M. M., Barta, M., Goodman, M., Cox, C. E., \& Kip, K. E. (2009). Randomized controlled trial of Mindfulness-Based Stress Reduction (MBSR) for survivors of breast cancer. Psycho-Oncology, 18(12), 1261-1272. https://doi.org/10.1002/pon.1529

Lengacher, C. A., Reich, R. R., Paterson, C. L., Ramesar, S., Park, J. Y., Alinat, C., Johnson-Mallard, V., Moscoso, M., Budhrani-Shani, P., Miladinovic, B., Jacobsen, P. B., Cox, C. E., Goodman, M., \& Kip, K. E. (2016). Examination of broad symptom improvement resulting from Mindfulness-Based Stress Reduction in breast cancer survivors: A randomized controlled trial. Journal of Clinical Oncology: Official Journal of the American Society of Clinical Oncology, 34(24), 2827-2834. https://doi.org/10.1200/JCO.2015.65.7874

Lengacher, C. A., Reich, R. R., Paterson, C. L., Shelton, M., Shivers, S., Ramesar, S., Pleasant, M. L., Budhrani-Shani, P., Groer, M., Post-White, J., JohnsonMallard, V., Kane, B., Cousin, L., Moscoso, M. S., Romershausen, T. A., \& Park, J. Y. (2019). A large randomized trial: Effects of Mindfulness-Based Stress Reduction (MBSR) for breast cancer (BC) survivors on salivary cortisol and IL-6. Biological Research for Nursing, 21(1), 39-49. https://doi.org/10.1177/1099800418789777

Leykin, Y., Thekdi, S. M., Shumay, D. M., Muñoz, R. F., Riba, M., \& Dunn, L. B. (2012). Internet interventions for improving psychological well-being in psycho-oncology: Review and recommendations. Psycho-Oncology, 21(9), 1016-1025. https://doi.org/10.1002/pon.1993

Liberati, A., Altman, D. G., Tetzlaff, J., Mulrow, C., Gøtzsche, P. C., Ioannidis, J. P., Clarke M., Devereaux P.J., Kleijnen J., \& Moher, D. (2009). The PRISMA statement for reporting systematic reviews and meta-analyses of studies that evaluate health care interventions: Explanation and elaboration. Journal of Clinical Epidemiology, 62(10), e1-e34. https://doi.org/10.1016/j.jclinepi.2009.06.006

Ma, Y., She, Z., Siu, A. F., Zeng, X., \& Liu, X. (2018). Effectiveness of online mindfulness-based interventions on psychological distress and the mediating role of emotion regulation. Frontiers in Psychology, 9,2090. https://doi.org/10.3389/fpsyg.2018.02090

Mehta, V., Goel, S., Kabarriti, R., Cole, D., Goldfinger, M., Acuna-Villaorduna, A., Pradhan K., Thota, R., Reissman, S., Sparano, J.A., Gartrell, B.A., Smith, R.V, Ohri, N., Garg, M., Racine, A.D., Kalnicki, S., Perez-Soler R., Halmos, B., \& Verma, A. (2020). Case fatality rate of cancer patients with COVID-19 in a New York hospital system. Cancer Discovery, 10(7), 935-941. DOI: 10.1158/2159-8290.CD-20-0516

Messer, D., Horan, J. J., Larkey, L. K., \& Shanholtz, C. E. (2019). Effects of internet training in mindfulness meditation on variables related to cancer recovery. Mindfulness, 10(10), 2143-2151. https://doi.org/10.1007/s12671-019-01182-y

Metin, Z. G., Karadas, C., Izgu, N., Ozdemir, L., \& Demirci, U. (2019). Effects of progressive muscle relaxation and mindfulness meditation on fatigue, coping styles, and quality of life in early breast cancer patients: An assessor blinded, three-arm, randomized controlled trial. European Journal of Oncology Nursing, 42,116-125. https://doi.org/10.1016/j.ejon.2019.09.003

Mikolasek, M., Berg, J., Witt, C. M., \& Barth, J. (2018). Effectiveness of mindfulness- and relaxation-based ehealth interventions for patients with medical conditions: A systematic review and synthesis. International Journal of Behavioral Medicine, 25(1), 1-16. https://doi.org/10.1007/s12529-017-9679-7

Morrill, E. F., Brewer, N. T., O'Neill, S. C., Lillie, S. E., Dees, E. C., Carey, L. A., \& Rimer, B. K. (2008). The interaction of post-traumatic growth and post-traumatic stress symptoms in predicting depressive symptoms and quality of life. Psycho-Oncology: Journal of the Psychological, Social and Behavioral Dimensions of Cancer, 17(9), 948-953. https://doi.org/10.1002/pon.1313

Ngamkham, S., Holden, J. E., \& Smith, E. L. (2019). A systematic review: Mindfulness intervention for cancer-related pain. Asia-Pacific Journal of Oncology Nursing, 6(2), 161-169. https://doi.org/10.4103/apjon.apjon_67_18

Nissen, E. R., O'Connor, M., Kaldo, V., Højris, I., Borre, M., Zachariae, R., \& Mehlsen, M. (2020). Internet-delivered mindfulness-based cognitive therapy for anxiety and depression in cancer survivors: A randomized controlled trial. Psycho-Oncology, 29(1), 68-75. https://doi.org/10.1002/pon.5237

Parodi, S. M., \& Liu, V. X. (2020). From containment to mitigation of COVID-19 in the US. Jama, 323(15), 1441-1442. doi:10.1001/jama.2020.3882 
Russell, L., Ugalde, A., Milne, D., Krishnasamy, M., Austin, D. W., Chambers, R., Orellana, L. \& Livingston, P. M. (2018). Feasibility of an online mindfulness-based program for patients with melanoma: Study protocol for a randomised controlled trial. Trials, 19(1), 1-11. https://doi.org/10.1186/s13063018-2575-x

Schell, L. K., Monsef, I., Wöckel, A., \& Skoetz, N. (2019). Mindfulness-based stress reduction for women diagnosed with breast cancer. The Cochrane Database of Systematic Reviews, 3(3), CD011518. https://doi.org/10.1002/14651858.CD011518.pub2

Simard, S., Thewes, B., Humphris, G., Dixon, M., Hayden, C., Mireskandari, S., \& Ozakinci, G. (2013). Fear of cancer recurrence in adult cancer survivors: A systematic review of quantitative studies. Journal of Cancer Survivorship: Research and Practice, 7(3), 300-322. https://doi.org/10.1007/s11764-013-0272-z

Spijkerman, M. P., Pots, W. T., \& Bohlmeijer, E. T. (2016). Effectiveness of online mindfulness-based interventions in improving mental health: A review and meta-analysis of randomised controlled trials. Clinical Psychology Review, 45,102-114. https://doi.org/10.1016/j.cpr.2016.03.009

Subnis U.B., Farb N.A., Piedalue K.L., Speca M., Lupichuk S., Tang P.A., Faris P., Thoburn M., Saab B.J. \& Carlson L.E. (2020). A smartphone app-based mindfulness intervention for cancer survivors: protocol for a randomized controlled trial. JMIR Research Protocols, 9(5), e15178. doi: 10.2196/15178

Tauber, N. M., O'Toole, M. S., Dinkel, A., Galica, J., Humphris, G., Lebel, S., Maheu, C., Ozakinci, G., Prins, J., Sharpe, L., Smith, A. B., Thewes, B., Simard, S., \& Zachariae, R. (2019). Effect of psychological intervention on fear of cancer recurrence: A systematic review and meta-analysis. Journal of Clinical Oncology: Official Journal of the American Society of Clinical Oncology, 37(31), 2899-2915. https://doi.org/10.1200/JCO.19.00572

Trevino, K. M., Raghunathan, N., Latte-Naor, S., Polubriaginof, F. C., Jensen, C., Atkinson, T. M., Emard, N., Seluzicki, C.M., Ostroff J.S. \& Mao, J. J. (2021). Rapid deployment of virtual mind-body interventions during the COVID-19 outbreak: feasibility, acceptability, and implications for future care. Supportive Care in Cancer, 29(2), 543-546. https://doi.org/10.1007/s00520-020-05740-2

Ueda, M., Martins, R., Hendrie, P. C., McDonnell, T., Crews, J. R., Wong, T. L., McCreery, B., Jagels, B., Crane, A., Byrd, D. R., Pergam, S. A., Davidson, N. E., Liu, C., \& Stewart, F. M. (2020). Managing cancer care during the COVID-19 pandemic: Agility and collaboration toward a common goal. Journal of the National Comprehensive Cancer Network: JNCCN, 1-4. Advance online publication. https://doi.org/10.6004/jnccn.2020.7560

Xie, C., Dong, B., Wang, L., Jing, X., Wu, Y., Lin, L., \& Tian, L. (2020). Mindfulness-Based Stress Reduction can alleviate cancer- related fatigue: A metaanalysis. Journal of Psychosomatic Research, 130, 109916. https://doi.org/10.1016/j.jpsychores.2019.109916

Xunlin, N. G., Lau, Y., \& Klainin-Yobas, P. (2020). The effectiveness of mindfulness-based interventions among cancer patients and survivors: A systematic review and meta-analysis. Supportive Care in Cancer, 28(4), 1563-1578. https://doi.org/10.1007/s00520-019-05219-9

Zernicke, K. A., Campbell, T. S., Speca, M., McCabe-Ruff, K., Flowers, S., Dirkse, D. A., \& Carlson, L. E. (2013). The eCALM Trial-eTherapy for cancer appLying mindfulness: online mindfulness-based cancer recovery program for underserved individuals living with cancer in Alberta: Protocol development for a randomized wait-list controlled clinical trial. BMC Complementary and Alternative Medicine, 13, 34. https://doi.org/10.1186/1472-6882-13-34

Zernicke, K. A., Campbell, T. S., Speca, M., McCabe-Ruff, K., Flowers, S., \& Carlson, L. E. (2014). A randomized wait-list controlled trial of feasibility and efficacy of an online mindfulness-based cancer recovery program: The eTherapy for cancer applying mindfulness trial. Psychosomatic Medicine, 76(4), 257267. https://doi.org/10.1097/PSY.0000000000000053

Zernicke, K.A., Campbell, T.S., Speca, M., Kelley, M., Ruff, M., Flowers, E., Tamagawa, R. \& Carlson, L.E., et al. (2016) The eCALM Trial: eTherapy for Cancer Applying Mindfulness. Exploratory analyses of the associations between online Mindfulness-Based Cancer Recovery participation and changes in mood, stress symptoms, mindfulness, posttraumatic growth, and spirituality. Mindfulness, 7, 1071-1081. https://doi.org/10.1007/s12671-016-0545-5

Wolvers, M., Bruggeman-Everts, F. Z., Van der Lee, M. L., Van de Schoot, R., \& Vollenbroek-Hutten, M. M. (2015). Effectiveness, mediators, and effect predictors of internet interventions for chronic cancer-related fatigue: The design and an analysis plan of a 3 -armed randomized controlled trial. JMIR Research Protocols, 4(2), e77. https://doi.org/10.2196/resprot.4363

World Health Organization. (2020). Considerations for quarantine of individuals in the context of containment for coronavirus disease (COVID-19): Interim guidance, 19 March 2020 (No. WHO/2019-nCoV/IHR_Quarantine/2020.2). World Health Organization.

Young, A. M., Ashbury, F. D., Schapira, L., Scotté, F., Ripamonti, C. I., \& Olver, I. N. (2020). Uncertainty upon uncertainty: Supportive care for cancer and COVID-19. Supportive Care in Cancer: Official Journal of the Multinational Association of Supportive Care in Cancer, 28(9), $4001-4004$. https://doi.org/10.1007/s00520-020-05604-9 